\section{CASE REPORT}

\author{
J. Linn \\ J. Herms \\ M. Dichgans \\ H. Brückmann \\ G. Fesl \\ T. Freilinger \\ M. Wiesmann
}

\title{
Subarachnoid Hemosiderosis and Superficial Cortical Hemosiderosis in Cerebral Amyloid Angiopathy
}

\begin{abstract}
SUMmARY: Cerebral amyloid angiopathy (CAA) is an important cause of intracerebral hemorrhage. Its definite diagnosis still requires histopathologic demonstration of vascular amyloid. Thus, further improvement of noninvasive imaging methods would be desirable. Here we present 3 patients with histologically proved CAA, in which superficial cortical hemosiderosis and subarachnoid hemosiderosis were present in $\mathrm{T} 2{ }^{*}$-weighted MR images. Thus, we propose that these 2 findings might be valuable as noninvasive diagnostic markers for CAA.
\end{abstract}

C erebral amyloid angiopathy (CAA) is defined as the deposition of amyloid in the walls of cortical and leptomeningeal blood vessels. The most important clinical presentation of CAA is intracerebral hemorrhage $(\mathrm{ICH}),{ }^{1,2}$ but identifying CAA as the cause of $\mathrm{ICH}$ is challenging. According to the most widely used Boston diagnostic criteria, the definite diagnosis of CAA and the diagnosis of probable CAA with supporting pathologic findings still require the histopathologic demonstration of vascular amyloid as a criterion standard. ${ }^{3,4}$ Thus, further improvement of the sensitivity and specificity of noninvasive imaging methods would be desirable to increase the value of noninvasive diagnostic measures.

Here we report on 3 consecutive patients with histologically proved CAA who presented to our hospital between June 2002 and June 2006. On the basis of the imaging findings in these patients, we propose superficial cortical hemosiderosis and subarachnoid hemosiderosis as potentially useful new MR imaging criteria to facilitate the noninvasive diagnosis of CAA. In this context, we defined the term subarachnoid hemosiderosis as hemosiderosis in the subarachnoid space. Superficial cortical hemosiderosis is defined as linear residues of blood in the superficial layers of the cerebral cortex as opposed to the punctate subcortical microhemorrhages often described in CAA. ${ }^{5}$

\section{Case Reports}

\section{Patient 1}

A 70-year-old woman initially presented with slowly progressive aphasia and apraxia. MR imaging revealed a left parietal leptomeningeal lesion with signal hyperintensity on proton-attenuation (PD)weighted images, and a linear leptomeningeal contrast enhancement on contrast-enhanced T1-weighted images. T2*-weighted images were not performed at this time. Analysis of the CSF showed no pathologic findings.

One week later, the patient experienced acute global aphasia and

Received April 20, 2007; accepted after revision June 29

From the Department of Neuroradiology (J.L., H.B., G.F., M.W.), Zentrum fur Neuropathologie und Prionforschung (ZNP) (J.H.), and the Department of Neurology (M.D., T.F.), University of Munich, Munich, Germany.

Please address correspondence to Jennifer Linn, Department of Neuroradiology, University of Munich, Grosshadern, Marchioninistr 15, 81377 Munich, Germany; e-mail: linn@nrad.de DOI 10.3174/ajnr.A0783 severe apraxia. CT revealed an acute left temporal $\mathrm{ICH}$, adjacent to the leptomeningeal lesion. MR imaging also demonstrated the acute left temporal ICH. In addition, T2*-weighted images depicted superficial cortical hemosiderosis in the left parietal lobe (Fig. 1), and subarachnoid hemosiderosis in the left frontal lobe. CT did not show any evidence of acute subarachnoid hemorrhage in the left parietal lobe, but in the left frontal lobe. Digital subtraction angiograms did not show any arteriovenous malformation, dural arteriovenous shunts, or sinus or venous thrombosis.

Surgical evacuation of the left temporal ICH including open biopsy of the left parietal meninges was performed. The histopathologic examination revealed beta A4-positive CAA with involvement of the meningeal as well as the cerebral vessels and brain parenchyma.

\section{Patient 2}

A 69-year-old man presented at an outpatient clinic with a severe headache, visual disturbances, and nausea lasting 3 weeks. Imaging revealed a left frontal ICH. On admission to our hospital, the patient presented with left-sided hemiplegia. MR imaging revealed an acute ICH in the right anterior temporal lobe and a subacute ICH in the left frontal lobe. In addition, $\mathrm{T} 2^{\star}$-weighted gradient-echo images demonstrated left frontal subarachnoid hemosiderosis, and left parietal superficial cortical hemosiderosis (distant from the acute macrohemorrhages [Fig 2]). Surgical resection of the right temporal ICH via

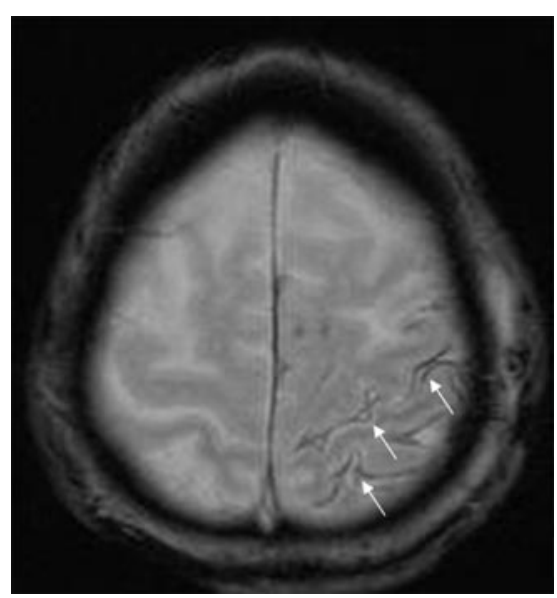

Fig 1. Patient 1, a 70-year-old woman with global aphasia and severe apraxia. T2* weighted image demonstrates superficial cortical hemosiderosis in the left parietal cortex (arrows). 

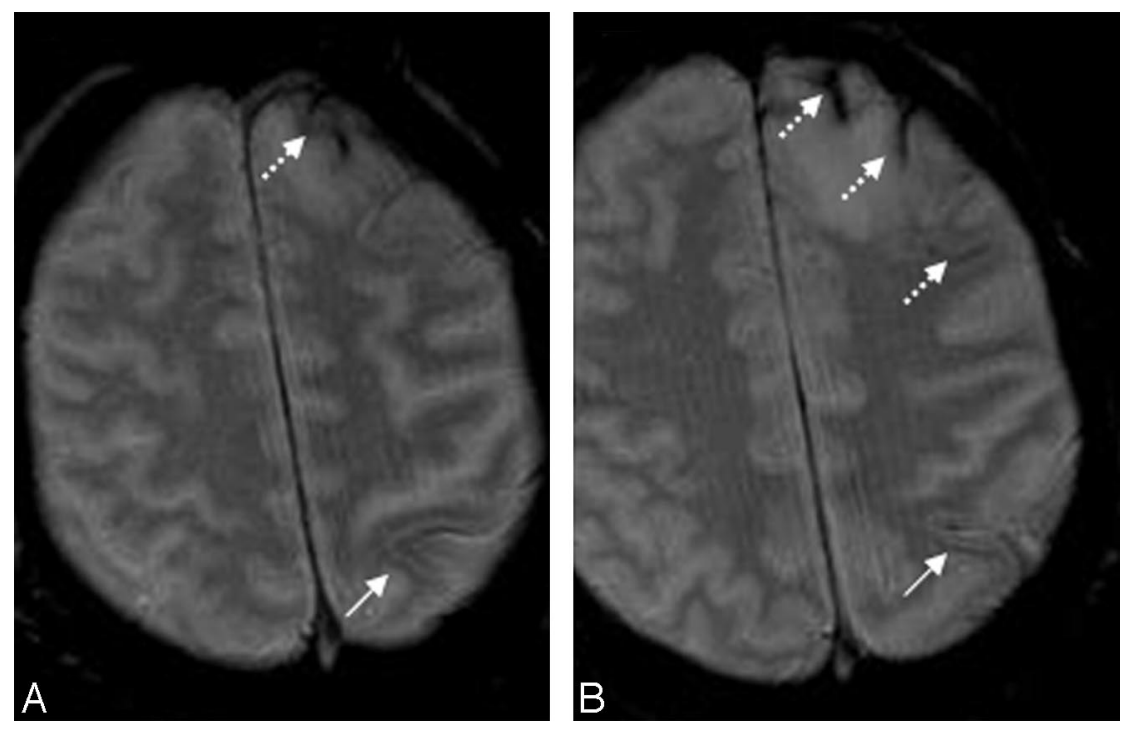

Fig 2. Patient 2, a 69-year-old man with severe headache, visual disturbances, and nausea. $A, B, T 2^{*}$-weighted images depict left frontal subarachnoid hemosiderosis (dotted arrows) and left parietal superficial cortical hemosiderosis (arrows).
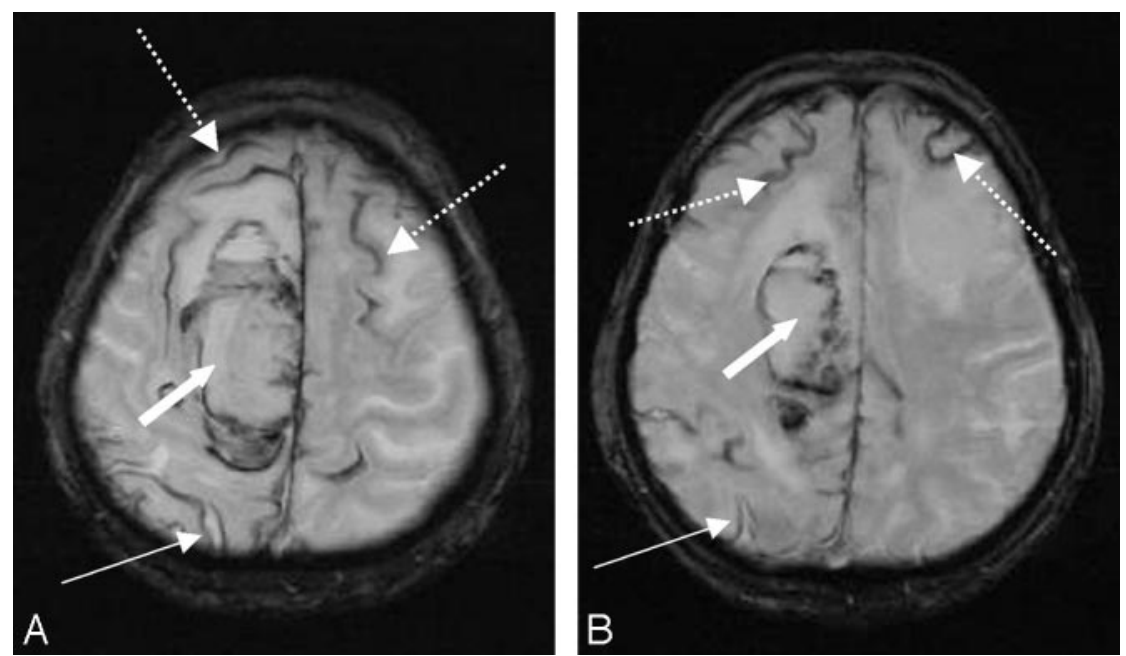

Fig 3. Patient 3 , a 72-year-old woman with histopathologically proved CAA and a large hyperacute right frontal intracerebral macrohemorrhage (thick arrows). $A, B, \mathrm{~T} 22^{*}$-weighted images demonstrate the hyperacute lobar hemorrhage (thick arrows), subarachnoid hemosiderosis (dotted lines), and superficial cortical hemosiderosis (thin arrows).

right temporal trepanation and histopathologic evaluation of the resected material revealed CAA.

\section{Patient 3}

A 72-year-old woman with a history of breast cancer presented with an acute left-sided, brachiofacial hemiparesis, somnolence (Glasgow Coma Scale of 12), and a gaze deviation to the right side. According to her husband, the patient had experienced a right frontal ICH 3 years previously and had had some deficits in short-term memory since then.

Unenhanced cranial CT demonstrated an acute right frontal ICH. MR imaging revealed a hyperacute, right frontal lobar hemorrhage with signal isointensity on T1-weighted images. In addition to the hyperacute ICH, T2*-weighted gradient-echo images showed subarachnoid hemosiderosis and superficial cortical hemosiderosis in the right frontal and parietal lobe, as well as in the left frontal lobe (Fig 3). As the PD-weighted MR images did not show any hyperintensity in the subarachnoid space in the respective regions, the hypointensity in the $\mathrm{T} 2{ }^{\star}$-weighted images was not caused by an acute subarachnoid hemorrhage. Instead, it corresponds to chronic subarachnoid hemosiderosis. The right frontal lesion did not show any abnormal contrast enhancement, and contrast-enhanced T1-weighted images did not reveal additional contrast-enhancing intracerebral lesions. Thus, there was no evidence of intracerebral metastasis of the breast cancer.
Evacuation of the right frontal ICH via a right parietal trepanation and subsequent histopathologic examination revealed CAA as the underlying cause of the ICH.

\section{Discussion}

Here we present the imaging findings of 3 patients with histologically proved CAA in which we observed superficial cortical hemosiderosis and subarachnoid hemosiderosis on $\mathrm{T}_{2}^{*}$ weighted images.

Secondary acute subarachnoid hemorrhage has been described in CAA in cases in which an ICH reaches the cortical surface. ${ }^{6}$ It has been speculated that CAA can cause primary subarachnoid hemorrhage as well, but data supporting this hypothesis are still sparse. ${ }^{6,7}$ We found chronic subarachnoid hemosiderosis at sites distant from the localization of acute ICH in 2 of our 3 patients. These observations indicate that these patients had clinically occult subarachnoid hemorrhages and that subarachnoid hemorrhage in CAA cannot only occur as an epiphenomenon if a lobar ICH reaches the cortical surface, ${ }^{6}$ but also as a primary hemorrhagic manifestation of CAA. This has also been suggested in cases of familial leptomeningeal amyloidosis. ${ }^{8,9}$ A neuropathologic study by Takeda et $\mathrm{al}^{10}$ further supports this hypothesis, as it shows that in some 


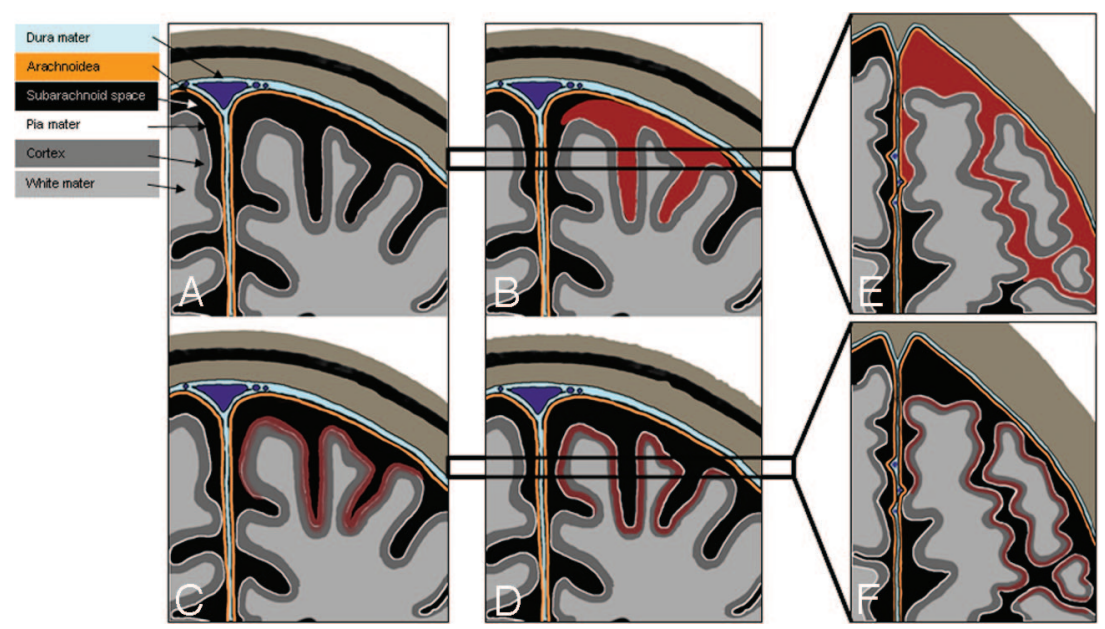

Fig 4. A-F, Schematic drawings illustrating subarachnoid hemosiderosis and superficial cortical hemosiderosis. $A-D$, Coronal schematic drawings, illustrating the time-dependent development of subarachnoid hemosiderosis and superficial cortical hemosiderosis (black, subarachnoid space; white line, pia mater; orange line, arachnoid layer; light blue, dura mater). E, F, Horizontal schematic drawings corresponding to the areas marked with black bars in $B$ and $C$, demonstrating the visual appearance of subarachnoid hemosiderosis and superficial cortical hemosiderosis on axial sections. A, Normal appearance of the subarachnoid space. $B, E$, Subarachnoid hemorrhage (red) presenting as a linear signal intensity in the subarachnoid space $(E)$. $C$, Residues of blood penetrating the pia mater and deposit in the superficial layers of the cerebral cortex. D,F, Superficial cortical hemosiderosis, defined as linear residues of blood in the superficial layers of the cerebral cortex (dark red). Superficial cortical hemosiderosis typically has a bilinear "tracklike" appearance on axial sections, caused by the signal intensity of the normal-appearing subarachnoid space in the middle, which is bordered bilaterally by linear deposits of hemosiderin in the superficial layers of the adjacent cortex $(F)$

cases of CAA the primary hemorrhage occurs in the subarachnoid space.

Besides subarachnoid hemosiderosis, we observed linear superficial cortical hemosiderosis, defined as linear blood residues in the superficial cortical layers of the brain on the T2*weighted images in our patients. In accordance with our findings, Kumar et $\mathrm{al}^{11}$ identified 1 patient in their study on superficial siderosis of the CNS who had a history of supratentorial intraparenchymal hemorrhages caused by CAA, in whom superficial deposits of hemosiderin were present over the cerebral convexities, but not in the posterior fossa, which is the typical localization of the hemosiderosis observed in superficial siderosis of the central nervous system. ${ }^{12}$

The superficial cortical hemosiderosis observed in our patients could have been caused by a primary bleeding in the superficial cortical layers, but it has been shown in animal models with experimental siderosis that repeated bleeding in the subarachnoid space leads to deposition of hemosiderin in the subpial layer of the brain. ${ }^{13}$ Thus, we propose the same mechanism as the underlying pathogenesis of the linear superficial cortical hemosiderosis that we observed in our patients. The schematic drawing in Fig 4 illustrates the development and imaging appearance of subarachnoid hemosiderosis and superficial cortical hemosiderosis.

In patient 1 , we observed a leptomeningeal contrast enhancement in a cortical region that was affected by CAA. Although this finding has been described previously in patients with familial amyloidosis, ${ }^{14}$ its detailed pathologic cause remains unclear.

On the basis of the imaging findings in the patients presented here, we propose superficial cortical hemosiderosis and subarachnoid hemosiderosis as potentially useful MR imaging criteria to facilitate the noninvasive diagnosis of CAA. Prospective studies with a larger number of cases are necessary to systematically assess the value of these MR signs in diagnostic settings.

\section{Acknowledgment}

We thank Ms. J. Benson for copyediting the manuscript.

\section{References}

1. Vinters HV. Cerebral amyloid angiopathy. A critical review. Stroke 1987;18:311-24

2. Qureshi AI, Tuhrim S, Broderick JP, et al. Spontaneous intracerebral hemorrhage. $N$ Engl J Med 2001;344:1450-60

3. Greenberg SM, Rebeck GW, Vonsattel JP, et al. Apolipoprotein E epsilon 4 and cerebral hemorrhage associated with amyloid angiopathy. Ann Neurol 1995;38:254-59

4. Knudsen KA, Rosand J, Karluk D, et al. Clinical diagnosis of cerebral amyloid angiopathy: validation of the Boston criteria. Neurology 2001;56:537-39

5. Greenberg SM, Finklestein SP, Schaefer PW. Petechial hemorrhages accompanying lobar hemorrhage: detection by gradient-echo MRI. Neurology 1996;46:1751-54

6. Yamada M, Itoh Y, Otomo E, et al. Subarachnoid hemorrhage in the elderly: a necropsy study of the association with cerebral amyloid angiopathy. J Neurol Neurosurg Psychiatry 1993;56:543-47

7. Ohshima T, Endo T, Nukui H, et al. Cerebral amyloid angiopathy as a cause of subarachnoid hemorrhage. Stroke 1990;21:480-83

8. Jin K, Sato S, Takahashi T, et al. Familial leptomeningeal amyloidosis with a transthyretin variant Asp18Gly representing repeated subarachnoid hemorrhages with superficial siderosis. J Neurol Neurosurg Psychiatry 2004;75:1463-66

9. Garzuly F, Vidal R, Wisniewski T, et al. Familial meningocerebrovascular amyloidosis, Hungarian type, with mutant transthyretin (TTR Asp18Gly) [published erratum appears in Neurology 1997;48:1143]. Neurology 1996;47:1562-67

10. Takeda S, Yamazaki K, Miyakawa T, et al. Subcortical hematoma caused by cerebral amyloid angiopathy: does the first evidence of hemorrhage occur in the subarachnoid space? Neuropathology 2003;23:254-61

11. Kumar N, Cohen-Gadol AA, Wright RA, et al. Superficial siderosis [published erratum appears in Neurology 2006;67:1528]. Neurology 2006;66:1144-52

12. Fearnley JM, Stevens JM, Rudge P. Superficial siderosis of the central nervous system. Brain 1995;118:1051-66

13. Koeppen AH, Dickson AC, Chu RC, et al. The pathogenesis of superficial siderosis of the central nervous system. Ann Neurol 1993;34:646-53

14. Urban PP, Hertkorn C, Schattenberg JM, et al. Leptomeningeal familial amyloidosis: A rare differential diagnosis of leptomeningeal enhancement in MRI. J Neurol 2006;253:1238-40 